\title{
MiR-155 acts as an inhibitory factor in atherosclerosis-associated arterial pathogenesis by down-regulating NoxA1 related signaling pathway in $\mathrm{ApoE}^{-/}$mouse
}

\author{
Yu Tang", Haoming Song", Yuqin Shen", Yian Yao, Yunan Yu, Guolian Wei, Bangxiang Long, \\ Wenwen Yan \\ Department of Cardiology, Tongji Hospital affiliated to Tongji University, Shanghai, China \\ Contributions: (I) Conception and design: W Yan, Y Tang; (II) Administration support: None; (III) Provision of study materials or patients: H Song, \\ Y Shen; (IV) Collection and assembly of data: Y Yao, Y Yu, G Wei, B Long; (V) Data analysis and interpretation: Y Tang, H Song, Y Shen; (VI) \\ Manuscript writing: All authors; (VII) Final approval of manuscript: All authors. \\ \#These authors contributed equally to this work. \\ Correspondence to: Wenwen Yan, MD, PhD. Department of Cardiology, Tongji Hospital affiliated to Tongji University, 389 Xincun Rd, Putuo District, \\ Shanghai 200065, China. Email: tj-yww@163.com.
}

Background: To investigate the protective efficacy of miR-155 on down regulating NADPH oxidase isoform subunit A1 (NoxA1) gene expression, resulting in inhibition of VSMC migration and over proliferation and thus ameliorating the progression of arterial atherosclerosis in AS mouse model. Therefore, to further explore the regulatory effect of miR-155 on neointima formation in AS and locate potential antiatherosclerosis target.

Methods: The mouse vascular aorta smooth muscle cell (MOVAS) was cultured and transfected with recombinant Pad2YFG adenovirus fluorescent vector with miR-155 fragment into 4 groups. Western blotting and RT-PCR were performed to identify the expression of NoxA1 under different circumstances. Fluorescence microscope was applied to observe the transfection rate of miR-155 into adenovirus. Twelveweek fatty food induced atherosclerotic $\mathrm{ApoE}^{-/-}$mouse model was established as host to accept miR-155 transfected adenovirus transplantation to observe its effect on VSMC in AS progression. Carotid and thoracic artery were extracted at 1 month after dosing. Distribution of miR-155 was quantified via expression levels of protein and RNA to detect NoxA1, Nox1, p47phox and NADPH expression. Immunohistochemistry, fluorescence imaging and other methods were performed in arteries section to compare the thickness of neointima and assess the severity of AS in each group.

Results: Luciferase reporter gene assay showed significant expression of miR-155 in mimic group indicating that miR-155 had target binding effect with NoxA1 gene. Western blotting and RT-PCR results both showed significantly decreased NoxA1 expression in miR-155 mimic group while increased with its inhibitor. The miR-155 distribution was observed varied at 1 month after in control, miR-155 mimic and inhibitor groups. The NoxA1, NADPH, Nox1 and pp47phox protein expression in VSMC was decreased in mimic group vs control and inhibitor groups $(\mathrm{P}<0.05)$; no significant difference of NADPH expression was observed in all groups. The NoxA1, Nox1 and p47phox gene expression in VSMC were both found reduced compared with those of control group at week $4(\mathrm{P}<0.05)$. Immunohistochemistry staining of artery frozen sections figured out that the thickness of neointima of carotid artery in miR-155 mimic group was significantly lower vs control and inhibitor groups $(\mathrm{P}<0.01)$ at week 4.

Conclusions: miR-155 played an important role in NoxA1-related signaling pathway. miR-155 transfection into VSMC may have anti-inflammatory regulatory effect on NoxA1 expression in vivo and resulting in amelioration of atherosclerotic lesion in AS mouse model. In summary, miR-155 specifically plays in a negative feedback loop and demonstrates a protective role during atherosclerosis-associated VSMC proliferation and neointima formation through the miR-155-NoxA1-p47phox complex signaling pathway. 
Keywords: Atherosclerosis; miR-155; neointima; NoxA1

Submitted May 19, 2020. Accepted for publication Jul 31, 2020.

doi: $10.21037 /$ cdt-20-518

View this article at: http://dx.doi.org/10.21037/cdt-20-518

\section{Introduction}

Cardiovascular disease, still the leading cause of death in humans, has become a major public health problem in China, with its incidence rising by 46\% from 1990 to 2013 $(1,2)$. Coronary atherosclerotic disease (CAD) is the most common type of cardiovascular disease and its pathogenesis is arterial atherosclerosis (AS). AS is a condition caused by lipid-induced inflammation of the vessel wall of various cell types, such as endothelial cells and vascular smooth muscle cells (VSMC). On the basis of atheromatous plaque, the accumulation of foam cells and intimal VSMC thickening formed fiber fatty plaques, and softening plaque cap rupture inevitable, leading to acute thrombotic events such as the occurrence of myocardial infarction and stroke $(3,4)$. The high mortality and high morbidity have brought great harm to society and the economic burden. Therefore, it has become a global social and economic need to explore new approaches to developing joint intervention and treatment of atherosclerosis.

MicroRNAs (miRNAs), as a research hotspot in recent years, have emerged as key regulators of gene expression by repressing the target mRNA, which determines cell functions under both homeostatic and disease conditions, which can be used as new ideas to further interpret the above problems at the molecular level. Such small molecules bind to complementary mRNA molecules, resulting in gene silencing that affects protein synthesis and phenotypic changes. Therefore, the in-depth study of the important genes related to the proliferation and migration of VSMC and the targeted regulation of miRNA may provide new ideas for gene screening of arterial disease risk factors, prevention and treatment of atherosclerosis and the development of new targeted drugs.

Similar to various mRNAs in human body, miR-155 is a short non-coding RNA fragment composed of about 20 bases, encoded by B-cell colony stimulating gene (Bic), and expressed in a variety of hematopoietic cells (5). Current studies have shown that miR-155 plays a role as a major regulator in a variety of biological processes, especially in immune function and tumorigenesis (6). Previous studies on miR-155 mainly focused on macrophagic foam degeneration and lipid metabolism, while few studies on smooth muscle cell proliferation and migration, another important cause of atherosclerosis. Previous studies observed that miR-155, which is implicated in proliferation and inflammation is involved in neointimal hyperplasia. Moreover, excessive vascular epithelial reactive oxygen species production by NADPH oxidase isoform subunit $\mathrm{A} 1$ (NoxA1) has been implicated in a number of disease states including atherosclerosis and species-specifically exists in VSMC of human and rodents. It will be a novel idea to explore the correlation between miR-155 and NoxA1 and the concomitant regulatory effect on VSMC proliferation.

The Nox1-based NADPH oxidase contains regulatory cytosolic components p47phox, NoxA1 and p47phox are essential for NADPH oxidase activity in VSMCs. Existed results indicate that NoxA1 is increased in aortas and atherosclerotic lesions from $\mathrm{ApoE}^{-/-}$mice. Moreover, NoxA1 expression is correlated with the progression of atherosclerotic lesions, and both NoxA1 and p47phox were present in advanced atherosclerotic lesions of human carotid arteries. These results indicated that it would be a promising way to reduce vascular lesion formation and its complications by targeting NoxA1 and its phox-related complex with specific therapeutic interventions (7).

Neointimal hyperplasia refers to proliferation and migration of VSMCs primarily in the tunica intima, resulting in the thickening of arterial walls and decreased arterial lumen space. While the inhibition of NoxA1 expression can reduce the production of thrombin-induced superoxide free radicals, and local NoxA1 overexpression can significantly promote neointimal hyperplasia in the carotid artery injury model of mice. Increased expression of NoxA1 was also observed in the aortic and atherosclerotic lesions of $\mathrm{ApoE}^{-/-}$mice $(8,9)$. NoxA1 was associated with the progression of early atherosclerotic lesions and with 67 phox in advanced carotid artery lesions. All the above studies suggest that targeted treatment or intervention of NoxA1, as an important factor affecting vascular wall lesions and smooth muscle cell proliferation, may reduce the formation of vascular lesions and related complications. Therefore, it 
will be a novel idea to explore the correlation between miR155 and NoxA1 and the concomitant regulatory effect on neointima formation and further impact on atherosclerosis progression.

We have presented the following article in accordance with the ARRIVE reporting checklist (available at http:// dx.doi.org/10.21037/cdt-20-518).

\section{Methods}

Experiments were performed under a project license (NO.: 81700316) granted by institutional ethics committee board of Tongji Hospital affiliated to Tongji University, in compliance with Chinese national guidelines for the care and use of animals.

\section{Cell culture and treatment}

Mouse vascular aortic smooth muscle cell line (MOVAS) was obtained from Cell bank of Chinese Academy of Sciences and maintained in DMEM (Gibco, USA). The cells were cultured at $37^{\circ} \mathrm{C}$ in an incubator containing $5 \%$ $\mathrm{CO}_{2}$ cells, and the culture medium was mixed with $10 \%$ fetal bovine serum (Gibco, USA) and 1\% penicillin. The cell growth state was observed under the inverted phase contrast microscope (Olympus, Japan), and the culture medium and culture were replaced in time.

\section{3'-UTR luciferase reporter assays}

The mechanism of choosing NoxA1 as the target gene of miR-155 was originated from the search of the miRNA database (www.mirbase.org) and found that the sequence of Mmu-miR-155 MI0000177 highly resembled as that of Hsa-miR-155. The sequence map was shown as follows:

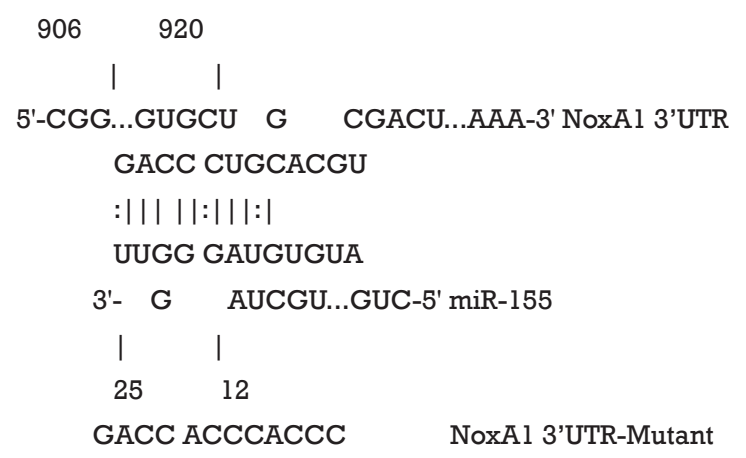

The prediction of miRNA target genes was performed using online software packages, including
MirDB, Targetscan, and PicTar. Design and synthesis of miR-155 mimics and inhibitor sequence ( $\left.5^{\prime}-3^{\prime}\right)$ : miR-155-mimics sense UUAAUGCUAAUUGUG AUAGGGGU, antisense CCCUAUCACAAUUA GCAUUAAUU; miR-155-inhibitor ACCCCUAUCA CAAUUAGCAUUAA.

miR-155 related sequences were constructed into plasmid and transfected into MOVAS for subsequent experiments. Experimental groups: miR-155 mimics + NoxAl, NC + NoxAl, miR-155 inhibitor +NoxAl, inhibitor + NC + NoxA1. Construction of NoxAl luciferase reporter gene vector included plasma transfection and luciferase reporter gene assay were conducted. Luciferase Reaction Substrate was fully decomposed with Luciferase Reaction Buffer to avoid repeated freezing-thawing. Luciferase Reaction Substrate II was mixed with Luciferase Reaction Buffer II at a ratio of 1:50. Mix $5 \times$ Cell Lysic Buffer with $d_{d H_{2}} \mathrm{O}$ in a 1:4 ratio. Added an appropriate amount of $1 \times$ Cell Lysic Buffer, fully lyse at room temperature for 10 minutes, take the supernatant for use. $100 \mu \mathrm{L}$ Luciferase Reaction Reagent was added to the test tube at equilibrium at room temperature, and $20 \mu \mathrm{L}$ cell lysates were added to the test tube to detect the activity of firefly luciferase reporter gene in the chemiluminescence apparatus. Luciferase Reaction Reagent II was added to the test tube to detect the luciferase activity in the chemiluminescence apparatus.

\section{RNA isolation and quantitative real-time PCR}

qPCR primers for miR-155, NoxAl and NADPH (5'3'): miR-155, TTAATGCTAATTGTGATAGGGGT; NoxAl-F, ACGCGAAGACTGGGACTCT; NoxAl-R AGCCCCTGTTAAAGTACATCCTA; NADPH-F, GATCCGGGTCCTCAGAGGTTT; NADPH-R, ATCAGGTGGTAGCATAGGCTT.

Experiments of cell or tissue RNA extraction were performed using the protocols suggested in the Trizol reagent specification (Invitrogen, 15596-018). qRT-PCR was performed by two-step PCR (Step OnePlus, Applied Biosystems).

\section{Western blotting analysis of NoxA1 and NADPH}

Protein expression of NoxAl and NADPH was detected by Western blotting. Membrane blocking and antibody incubation included 5\% skim milk powder blocked at room temperature for 1 hour. Primary antibody was diluted according to the instructions, and the antibody is diluted 
to the required concentration in the sealing solution, and the membrane was incubated at $4{ }^{\circ} \mathrm{C}$ overnight. Secondary antibody: the membrane incubated with primary antibody was washed with TBST for 3 times, each time for $10 \mathrm{~min}$. And then depending on the dosage, the membrane was then incubated at room temperature according to the diluted HRP labeled secondary antibody.

\section{Scratching assay to test inbibitory effect of miR-155 on MOVAS migration}

Use marker pen on the back of the 6-hole plate first and compare with a ruler to draw a horizontal line evenly, about every $0.5-1 \mathrm{~cm}$, across the hole. Each hole passes through at least 5 lines. Add about $5 \times 10^{5}$ cells, the number can be spread overnight. On the second day, use the pipette head to compare the ruler and hang down to the back of the horizontal scratches, head should be vertical, not tilted. Wash the cells with PBS for 3 times, remove the scratched cells, and add serum-free medium. Put it into a $37{ }^{\circ} \mathrm{C} 5 \% \mathrm{CO}_{2}$ incubator for culture. Sampling at 0,24 h and take photos.

\section{Construction of adenovirus containing fluorescent labeled miR-155}

After homologous recombination of miR-155 fragment with PAd2YFG adenovirus fluorescent expression vector, it was transferred into competent bacteria containing Adeasy-1 plasmid by electro transformation. Kanamycin resistant clones were screened by LB plate and transfected into package cells 293 after purification and amplification. When the growth reached confluence of $70-80 \%$, the 293 cells expressed green fluorescence and showed cytopathic signs after 4-5 days after addition of the virus solution. The cells and supernatant were both collected. $-70{ }^{\circ} \mathrm{C} / 37^{\circ} \mathrm{C}$ freezingthawing cycle was repeated for 4 times with 5 min each time. After the cells were completely lysed, the supernatant was centrifuged to harvest the virus. When the fourth generation VSMC of the above-mentioned cell line was grown to $70-80 \%$ fusion, the culture medium was discarded and the adenovirus suspension with miR-155 fragment was attached in $2 \mathrm{~h}$. The transfection rate of adenovirus VSMC was observed by fluorescence microscopy after $12 \mathrm{~h}$.

\section{High-fat diet atherosclerotic mouse model establishment and miR-155 transfected adenovirus injection}

Healthy 8-week SPF-level male ApoE ${ }^{-/-}$mice of body weigh 18 to $23 \mathrm{~g}$ were averagely divided into a high-fat diet group and an ordinary diet group. High-fat diet group was fed with $21 \%$ fat and $0.15 \%$ cholesterol for 12 weeks. The ordinary group was fed with regular chow. Few mice in each group were sacrificed by the end of 12 weeks and the abdominal aorta and coronary artery were extracted for pathological examination to verify the success of AS model establishment (10).

Adenovirus suspension with $\mathrm{miR}-155 \mathrm{mimic} / \mathrm{miR}-155$ inhibitor/vector control transfected with immunofluorescent markers was injected into the tail vein in a total volume of $200 \mu \mathrm{L}$, respectively. The number of copies was about $2 \times 10^{9}$ plaque-forming units (PFU)/day/mouse, and continuous injection was performed for 3 days (11).

\section{Fluorescent In situ hybridization (FISH) of miR-155 expression in carotid and thoracic artery}

The material on the slides was denatured at $80{ }^{\circ} \mathrm{C}$ for $5 \mathrm{~min}$, and then the probes were allowed to hybridize at $37{ }^{\circ} \mathrm{C}$ in a moist chamber overnight. The sense and antisense digoxigenin (DIG)-labeled miR-155 riboprobes were prepared after linearization. miR155 was hybridized with sense and antisense miR-155 riboprobes, as previously described (12). The hybridization temperature was $43{ }^{\circ} \mathrm{C}$ and the posthybridization wash in $0.13 \mathrm{SSC} / 0.1 \%$ sodium dodecyl sulfate was performed at $50{ }^{\circ} \mathrm{C}$. The hybridized riboprobes were detected using the Nucleic Acid Detection Kit (GenePharma, Shanghai, $\mathrm{CN}$ ) according to the manufacturer's protocol. The sequence of the miR-155 probe was 5'-ROXCAGCTGGTTGAAGGGGACCAAA-3'. The slides were washed as described before, mounted in Fluorescence Mounting Medium (DakoCytomation) and analyzed using a Leica DMBR fluorescence microscope. Figures were captured, using the same exposure time for all slides, via a Leica DC (polychrome) camera.

\section{Arterial neointima formation histological analysis and NoxA1 signaling pathway expression detection}

The established AS ApoE ${ }^{-/-}$mice (C57BL6 background) with miR-155 mimic, miR-155 inhibitor and vehicle control were sacrificed at 1 month after dosing, respectively. Mouse abdominal aortas and thoracic artery were fixed in $4 \%$ formaldehyde/phosphate buffered saline (PBS), cleaned of fatty tissue, and stained with $0.2 \%$ Oil Red O (ORO, O0625, Sigma), after staining with oil red 
for $10-15$ min in dark room, the neutral fat was stained as red. The thoracic aorta and aortic arch were then cut open longitudinally and pinned out flat, intimal side up, for computer-assisted planimetric analysis. Images were taken with a digital camera (Olympus UC30) mounted on a stereo microscope (Olympus SZX3). The ORO stained lesion was quantified as a percentage of total area with SigmaScan Pro 5 (Systat Software Inc) by an observer blinded to genotype.

For neointima sample (hematoxylin \& eosin) HE staining, after perfusion-fixation with $4 \%$ paraformaldehyde, tissues of carotid/thoracic arteries were embedded in tissue embedding medium, snap-frozen, and stored at $-80{ }^{\circ} \mathrm{C}$. Washed from low concentration to high concentration of alcohol as a dehydrating agent, gradually removed the water in the tissue block. The tissue block was then placed in xylene and the transparent tissue block was placed in the melted paraffin and placed in the melting wax box for heat preservation. The paraffin was immersed into the tissue block completely and then embedded. Samples were sectioned on a Leica cryostat $(6-7 \mu \mathrm{m})$ and placed on polyL-lysine-coated slides for further immunohistochemical analysis. The slices were stained in aqueous solution of hematoxylin for several minutes and dye with alcohol eosin for 2-3 minutes.

$\mathrm{ApoE}^{-/-}$AS mouse model was established after 12-week high-fat chow ( $\mathrm{N}=6 /$ group) and euthanized 4 weeks after ad-miR-155 injection. Carotid and thoracic artery were extracted, and total RNA was extracted using Trizol reagent. After DNAse I (Takara, Tokyo, Japan) treatment, RNA was reverse transcribed with reverse transcriptase (Takara, Tokyo, Japan). The expression of NoxA1, Nox1, NADPH and $\mathrm{p} 47$ phox was normalized to that of actin.

\section{Statistical analysis}

Experimental data are presented as the mean (error bars indicate SEM) for normally distributed data or median with interquartile range (error bars indicate minimum and maximum) for nonnormally distributed data. Based on all experiments if the distribution was normal in most of the experiments, a 2-tailed $t$-test was used. If the distribution of a variable was skewed, the 2-tailed Mann-Whitney test was used. To compare multiple groups, one-way ANOVA was used for normally distributed data, and a Kruskal-Wallis test with the Dunn post hoc test was used for nonnormally distributed data.

\section{Results}

\section{MiR-155 was closely targeted to NoxA1 in vitro}

To verify the inhibitory efficacy of miR-155 on the transcription factor of NoxA1 gene, luciferase reporter assay was conducted in five groups including miR-155 mimics + NoxAl, negative control (NC) + NoxAl, miR-155 inhibitor $+\mathrm{NoxAl}$, inhibitor-NC+NoxA1 and NoxA1 respectively to detect the luciferase activity of NoxA1 under the impact of miR-155. In consistent with the anticipation and the transduction of anti-miR-155, the luciferase activity of miR-155+NoxA1 group was significantly lower than that of NoxA1 and $\mathrm{NC}+\mathrm{NoxA} 1$ groups $(\mathrm{P}<0.05$ or $\mathrm{P}<0.01)$. There was no statistical difference of luciferase activity among the other four groups (Figure 1A,B).

\section{MiR-155 inbibited NoxA1 expression and MOVAS migration at $R N A$, protein, and cell levels}

RNA and protein were extracted from MOVAS transfected with miR-155 or its inhibitor. Four groups including miR-155 mimic, miR-155 inhibitor, inhibitor + NC and control were set to detect the expression level of NoxA1 and NADPH. RT-PCR results showed that the miR-155 group significantly inhibited the expression of NoxA1 and NADPH compared with the inhibitor and control groups since NoxA1 is NADPH oxidase isoform subunit A1, thus NADPH expression should be correlated with NoxA1 expression level.

RT-PCR results demonstrated similar tendency as that of Western blotting. miR-155 mimic group inhibited NoxA1 gene expression to the largest extent and had significant difference compared with other groups $(\mathrm{P}<0.01)$ (Figure 1C,D).

In vitro scratching assay indicated that after transfection miR-155 mimic, 155 inhibitor into VSMC, miR-155 group significantly inhibit/mitigate MOVAS migration compared with that of 155 inhibitor and control groups (Figure 1E).

\section{miR-155 targets on arterial VSMC and regulates the pathological progression in arterial atherosclerosis mouse model}

ApoE $^{-/-}$mouse AS model was well established after 12week high-fat diet with subsequent normal chow. The atherosclerotic plaques burden in the aortic arch and thoracic aorta compared between miR-155 mimic and 


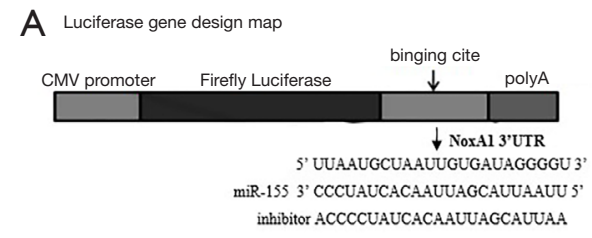

B Luciferase reporter assay on NoxA1 transcription

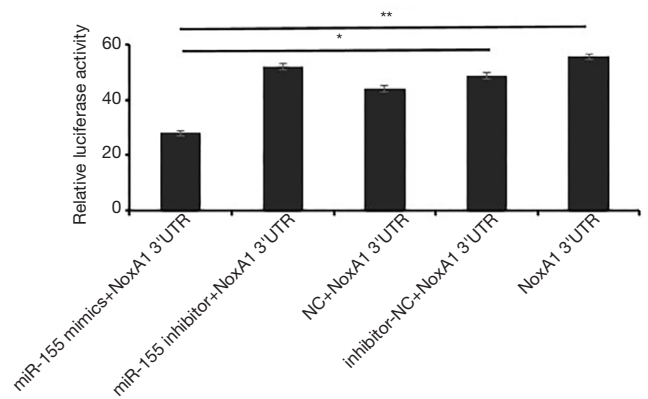

*, miR-155 mimic vs. three groups (NC + NoxA1, 155 inhibitor and inbibitost $+N C)(P<0.05)$; **, miR-155 vs. NoxA1 $(\mathrm{P}<0.01)$ (one-way ANOVA, $\mathrm{N}=3 /$ group)

\section{D}

Real-time PCR to detect NoxA1 and NADPH gene expression in MOVAS

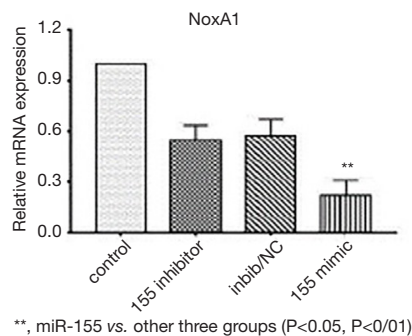

NADPH

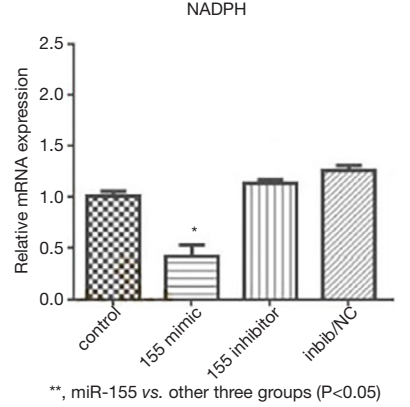

C Western blotting of NoxA1 expression affected by miR-155 in MOVAS
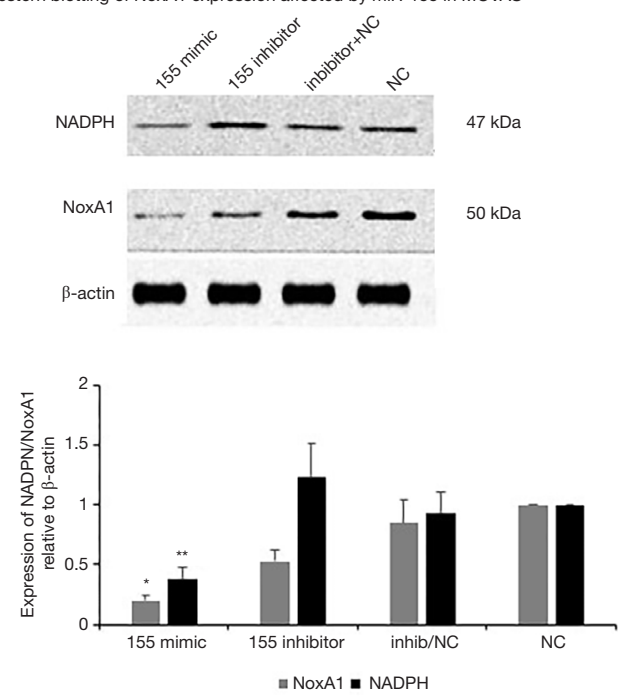

*, miR-155 vs. other three groups, $\mathrm{P}<0.05$; ${ }^{* *}$, miR-155 vs. inhibitor $+\mathrm{NC}$ and NC groups, $\mathrm{P}<0.05$

E
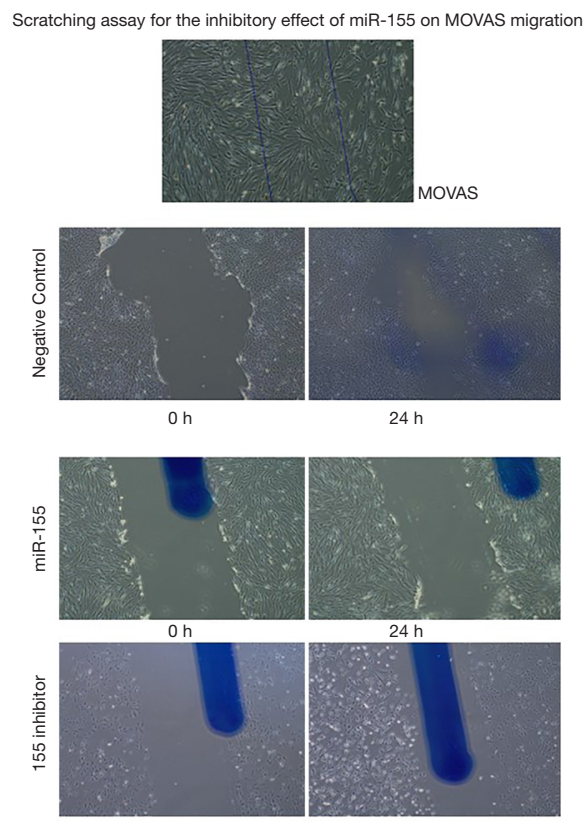

$24 \mathrm{~h}$

Figure 1 NoxA1 3'UTR and miR-155 binding cite map (Luciferase reporter assay rationale). Luciferase reporter gene assay on NoxA1 expression via miR-155 transcription was conducted and revealed the situation that miR-155 targeted on NoxA1 and inhibited its expression via luciferase activity decreasing in miR-155 mimics group which had significant difference from that of NC+NoxA1 and NoxA1 groups. No statistical difference found between and among other four groups except miR-155 mimics. NoxA1 expression was inhibited by miR-155 transfection in vitro. Protein of NoxA1 and NADPH was extracted from MOVAS and detected via Western blotting. There was significant inhibition of NoxA1 expression in miR-155 mimic group compared with NC and inhibitor + NC. NADPH also show inhibitory effect in miR-155 mimic group. The result of real-time PCR complied with that of Western blotting and showed the same inhibitory effect of miR155 mimic group on the expression of NoxA1. RNA samples were extracted from MOVAS transfected with miR-155, inhibitor or vector control. Similarly, NADPH also show inhibitory effect in miR-155 mimic group. The result of scratching assay in MOVAS transfected with miR-155. The miR-155 mimic group demonstrated inhibitory effect on MOVAS migration compared to that of 155 inhibitor and NC groups. Magnification in (E): $\times 50$. 


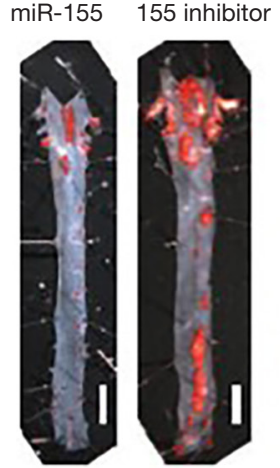

Aortic arch

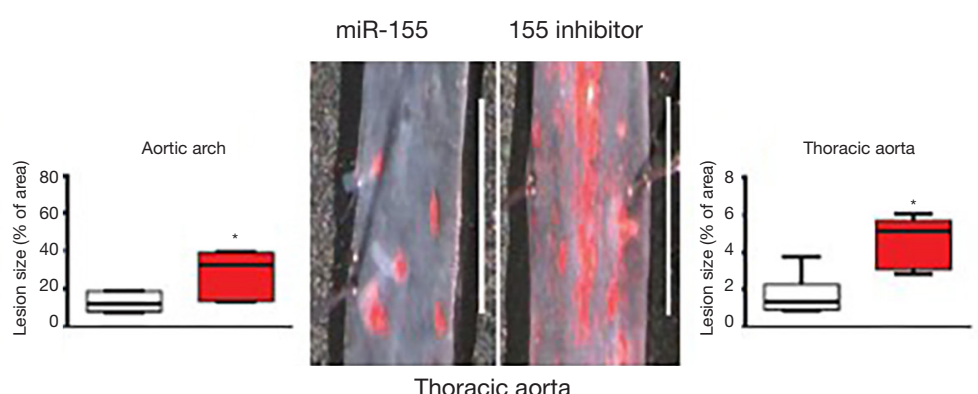

Figure $2 \mathrm{ApoE}^{-/-}$mouse AS plaque in aortic arch and thoracic aorta (miR-155 mimic vs. inhibitor). Macroscopic images showed the successful establishment of atherosclerotic model of ApoE ${ }^{-/-}$mice after 12-week high-fat diet feeding. Representative macro specimen demonstrated aortic arch and thoracic aortas stained with Oil Red O (ORO). The graph shows quantification of atherosclerosis burden and miR-155 mimic group expressed significant anti-atherosclerotic effect on AS progression than that of miR-155 inhibitor group. *, miR-155 vs. 155 inhibitor group, $\mathrm{P}<0.05$.

inhibitor group were shown as Figure $2(\mathrm{P}<0.05)$. The miR-155 expression was present in $30 \%$ to $90 \%$ of the carotid/thoracic aorta at 3 days and was absent 12 days after adenovirus injection. Adenovirus with green fluorescent protein (GFP) was detected (Figure $3 A$ ). In adenovirusmiRNA-155 reached arteries, the representative mouse of NC and miR-155 mimic group was sacrificed and the expression of antisense miR-155 RNA was demonstrated by in situ hybridization shown as Figure $3 B$.

\section{miR-155-regulated NoxA1 expression accelerates atherosclerosis progression in ApoE ${ }^{-/-}$mice atherosclerotic model mainly via neointima formation}

The $\mathrm{ApoE}^{-/-}$mice of miR-155 mimic, miR-155 inhibitor and vehicle control (NC) group were euthanized at 1 month (n=6/group). The isolated arteries were fixed with $4 \%$ paraformaldehyde in phosphate-buffered saline (PBS) via left ventricle injection. The areas of the right common carotid arteries, $15 \mathrm{~mm}$ around the bifurcation site, were paraffin-embedded and sectioned for ten continuous serial slides (5 $\mu \mathrm{m}$ in thickness) for hematoxylin and eosin (HE) staining. The miR-155 inhibitor and vehicle control groups demonstrated multi-layer VSMC migration and aggregation with narrower lumen and high intima/media ratio. While fewer VSMC could be found in miR-155 mimic group, resulting in much less neointima formation in the artery lumen and more total vessel area compared with inhibitor and vehicle control groups (Figure 4, $\mathrm{P}<0.05$ ).

Immunofluorescence images of aortas stained with anti-smooth muscle actin (SMA) antibody also showed the same trend that miR-155 mimic group characterized with normal VSMC accumulation while the other two groups had abnormal VSMC aggregation to accelerate the atherosclerosis (Figure 5).

\section{NoxA1 patbway was the direct target of miR-155}

The expression levels of major genes related to Nox signaling pathway were detected. The RNA expression levels of NoxA1, Nox1 and p47phox were statistically decreased in miR-155 mimic group compared with those of inhibitor and NC groups. NADPH expression decreased in miR-155 group but insignificant. miR-155 expression was definitely higher in miR-155 mimic group as anticipated and further verified the success of model establishment. Moreover, the SOD2, the main antioxidant product, which is specifically located in mitochondria, appeared to be significantly increased in miR-155 mimic group compared with that of 155 inhibitor group (Figure $6, \mathrm{P}<0.05, \mathrm{P}<0.01$ ). The Western blotting results showed the same trend as those of real-time PCR, indicating miR-155 played a pivotal role in regulating Nox signaling pathway in the pathogenesis of atherosclerosis.

\section{Discussion}

Atherosclerosis is one of the most common cardiovascular diseases worldwide, leading to severe cardiovascular complications with high mortality (13). This disease is 
A

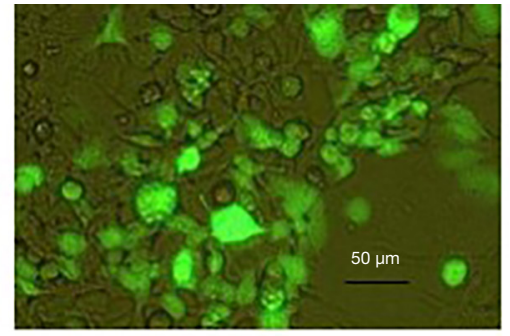

Adenovirus vector with GFP in vitro $(100 \times)$

B

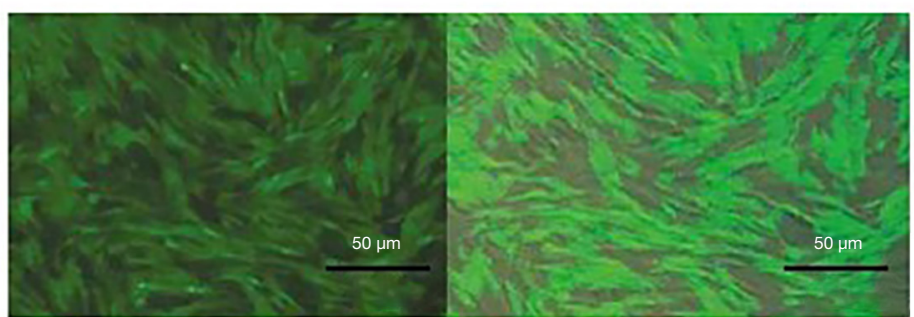

Mouse VSMC infected with NC vehicle (blank adenovinus vector) (L: brightfield, R: brightfield + fluorescence, $50 \times$ )

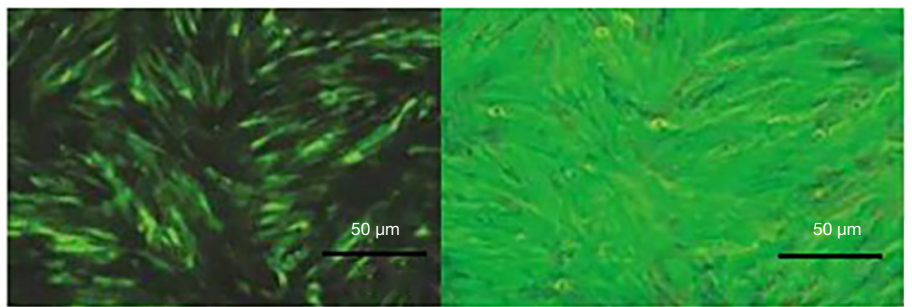

Mouse VSMC infected with adenovinus transfected with miR155 (L: brightfeld, R: brightfeld + fuorescence, $50 \times$ )

Figure 3 The infected adenoviruses were cultured in the supplemented medium for an additional 48 h before evaluation of green fluorescent protein (GFP) expression by FACS analysis. FISH was conducted to verify the successful transfection of adenovirus with miR-155 or vehicle vector into $\mathrm{ApoE}^{-/-}$mouse VSMC after tail vein injection for 3 days. The high ratio of transfection rate guaranteed the subsequent sample analysis.

characterized as chronic inflammation in response to lipid accumulation and foam cell formation. Most of foam cells have been revealed to be derived from macrophages, but VSMCs give rise to a considerable number of foam cells as well (14). VSMC and endothelial cells are the main effector cells that stimulate the vascular atherosclerotic reaction throughout the pathological process (15-17). The present study aims to elucidate the possible effector on VSMC and related arterial atherosclerotic pathogenesis.

Varied kinds of miR-155s have been reported to be related with inflammation and cardiovascular diseases (18). In present study, we focus on miR-155, a central regulator of immune responses that is highly expressed in activated immune cells in different signaling pathways, especially in ROS system. miR-155 is considered a pro-inflammatory miRNA, since one of its major targets is endogenous inhibitor of inflammatory signaling pathway $(19,20)$. It also induces foam formation and monocyte adhesion via miR-155/NOX2/NFkB-mediated ACAT-1 and VCAM-1 expressions in VSMCs (21).
In the early stage of atherosclerotic lesion, foam cell is a major hall-mark factor which also derived from VSMC. Therefore, a novel scope of miR-155 intervening specific gene expression in NOX/ROS related signaling pathway may give us a new overview on providing anti-atherosclerotic methods in the future. ROS is a critical contributor in the initiation and progression of atherosclerosis, and a major source for ROS in cardiovascular system is a family of NOX (22). Recent studies have shown that $\mathrm{NAD}(\mathrm{P}) \mathrm{H}$ oxidase-derived ROS in the brain is responsible for sympathetic activation and other super oxidation reaction. In the present study, we found that miR-155 targets NoxA1 very well and decreased NOXA1 expression and ROS related products in VSMCs both in vitro and in vivo, which was unique compared to its usual role in NFкB-ACAT-1/VCAM-1 mediated pathway $(23,24)$. The expression of NoxA1 in MOVAS of our study indicated that though there was slight amount of miR-155 existed in VSMC of control group, it would not significantly affect the expression of NoxA1, so did 

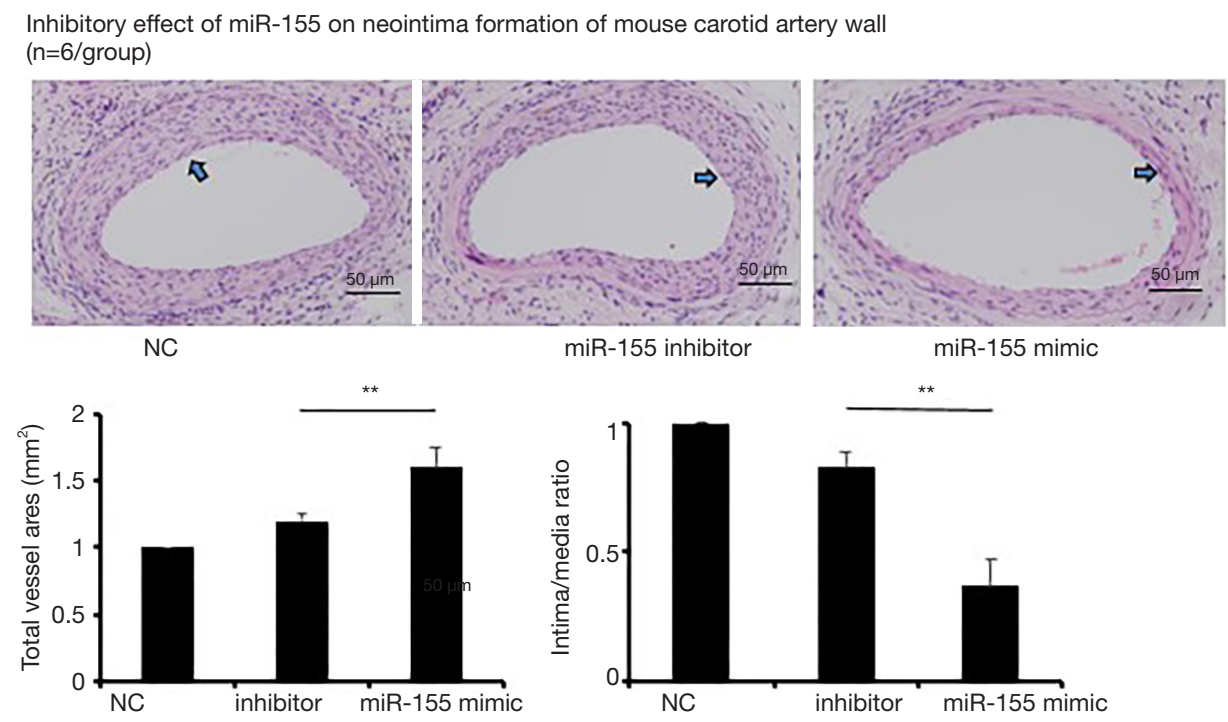

miR-155 mimic

Figure 4 One of the most significant characteristics in AS progression, neointima formation, was verified in carotid artery in vivo. HE staining of $\mathrm{ApoE}^{-/-}$AS model demonstrated the larger lumen volume and decreased neointima/media ratio in miR-155 mimic group compared with those of miR-155 inhibitor and NC groups. The stained blue nuclei corresponded to the accumulated/migrated VSMC. Blue arrowheads pointed at the area which was thickened neointima and indicting atherosclerotic progression. **, miR-155 mimic vs. 155 inhibitor, $\mathrm{P}<0.05$.

inhibitor and inhibitor + NC groups. However, exogenous mimic miR-155 could sharply inhibit the transcription of NoxA1 gene, which complies with the previous study that increased miR-155 relieves chronic inflammation by a negative feedback loop and plays a protective role during atherosclerosis-associated foam cell formation by signaling through the miR-155 related pathway (25).

NADPH oxidase is a key enzyme in the production of superoxide radicals $\left(\mathrm{O}_{2} \cdot^{-}\right)$and is associated with vascular pathology such as hypertension and atherosclerosis (26). NoxA1 is an important cellular regulatory component of $\mathrm{p} 47$ phox, and its overexpression is associated with the progression of atherosclerosis. NoxA1 and p47phox constitute the basic structure of Nox1-NADPH, which is unique to human and mouse VSMC $(7,27)$. Inhibition of NoxA1 expression can reduce the production of superoxide free radicals induced by thrombin. Local overexpression of NoxA1 can significantly promote neointimal hyperplasia in the model of carotid artery injury in mice. Increased expression of NoxA1 can also be seen in the aorta and atherosclerotic lesions of $\mathrm{ApoE}^{-/-}$mice (9). NoxA1 is associated with the progression of early atherosclerotic lesions, while it appears simultaneously with 47 phox in advanced carotid lesions in humans. All the above studies suggest that, as an important factor affecting vascular wall lesions and smooth muscle cell proliferation, targeted treatment or intervention of NoxA1 as a target may reduce the formation of vascular lesions and related complications, which complies with the main purport of the current study. In current in vitro experiments, we also verified the target effect of miR-155 on NoxA1 via miR-155 transfection into MOVAS to provide sufficient evidence for subsequent animal study.

In animal study, we proposed the specific impact of miR155 on NoxA1/NADPH/phox signaling pathway. Our data demonstrated for the first time an atheroprotective role of miR-155 and anti-inflammatory effects of miR-155 in vivo in hyperlipidemic conditions after successful establishment of AS in $\mathrm{ApoE}^{-/-}$mouse model. Firstly, miR-155 mimic group showed significant preventive effect on arterial neointima formation with relatively large lumen and low intima/media ratio compared with those of inhibitor and vehicle control. Secondly, the expression levels of NoxA1, NADPH, Nox1, and 47phox extracted from carotid/ thoracic arteries also indicated similar results as those in in vitro validation experiments. miR-155 significantly alleviated the expression of NoxA1, Nox1 and the component of NOX complex, 47 phox. Since NoxA1 is NADPH oxidase isoform subunit A1, thus NADPH expression also correlated with NoxA1 expression level as seen in this 

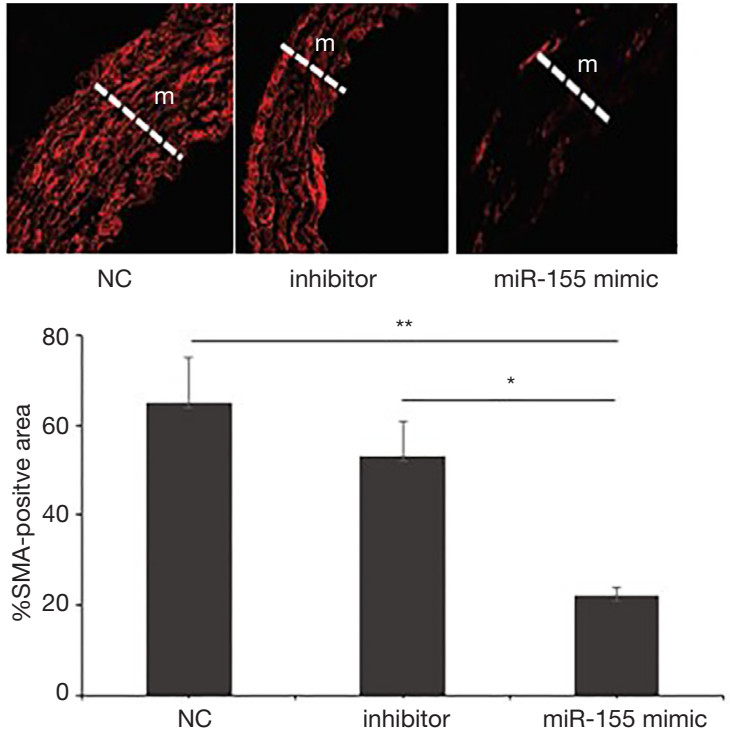

Figure 5 Representative immunofluorescence images of aortas stained with anti-smooth muscle actin (SMA) antibody (red, $\times 100$ ) in $\mathrm{ApoE}^{-/-}$AS mouse model. Graphs show quantification of VSMC content in the media (m) as either nucleus count or \% of SMApositive area. This method was used to verify that the formed neointima mainly consisted of VSMC and miR-155 mimic group showed mild accumulation in the media while moderate to severe VSMC accumulation was found in the media of miR-155 inhibitor and NC groups. *, miR-155 mimic vs. 155 inhibitor, $\mathrm{P}<0.01$; **, miR-155 mimic vs. NC, $\mathrm{P}<0.01$.

study. SOD2 decreasing in 155 inhibitor group further indicated the miR-155 may even have a bit anti-oxidation effect. More importantly, this AS $\mathrm{ApoE}^{-/-}$mouse help us extrapolate the possible expression pattern of miR-155. Since the specimens of arteries were extracted in 4 weeks after model establishment, which was in the early stage of atherosclerotic plaques formation, VSMC migration and accumulation. However, from our study the miR-155 group has shown significant anti-atherosclerotic efficacy on both neointima formation and blood vessel wall hyperplasia, which also complied with previous study results. All above results together with the meaningful inhibitory effect of miR-155 on MOVAS migration suggested that lack of miR-155 impaired the VSMC contractile phenotype and vasorelaxation by upregulating NoxA1/NADPH/ROS expression.

It can be inferred from our study that the function of miR-155 is highly dependent on the context and cell types (e.g., VSMC) with its early onset dynamic expression pattern and its suppressive effect on VSMC migration and multiple target genes, especially for NoxA1.

NoxA1 is an important regulatory hub that regulates the REDOX signaling pathway and the phenotype of VSMC. Depletion of this protein by retrovirus (e.g., adenovirus in current study) short hairpin RNA (shRNA) not only inhibited thrombase-induced reactive oxygen species (ROS), but also reduced cell proliferation (28). These studies have shown that the removal of NoxA1 can effectively inhibit the formation of diet-induced atherosclerosis in mice, thereby inhibiting the VSMCspecific NADPH oxidase to a certain extent. At present, NoxA1, as an important protein in ROS system, has gradually been known to control the negative effects of VSMC proliferation and vascular diseases, but the exact mechanism of regulating the expression of this protein is still unclear, the special effect of miR-155 may bring an effective way to block this pathogenic link. Our current RT-PCR results of mouse AS arteries demonstrated the fact that NoxA1 and Nox1 expression seemed to increase in atherosclerotic lesions suggesting that NoxA1 expression is fine-tuned to different pathophysiological stimuli like long-term high-fat diet. In this context, it elucidates a novel mechanism for NoxA1-regulated Nox1 activation in mouse VSMC but could be alleviated by addition of miR-155.

Therefore, it was speculated that the regulatory effect of miR-155 on NoxA1 was cleavage and inhibition of translation, that is, the level change of miR-155 was negatively correlated with the expression of target genes and downstream protein translation, which provided an important prediction and theoretical evidence for the possible results of preliminary experiments.

In conclusion, in this hyperlipidemic mouse model, miR-155 acts as an anti-inflammatory, atheroprotective microRNA. Additionally, apart from a confirmed role in lymphoid cell development, our in vitro and in vivo studies show a crucial role of miR-155 in NoxA1/47phox complex/ROS signaling pathway against AS formation and progressing. It would be intriguing to explore whether a similar phenomenon occurs in regard to NoxA1/p67phoxdependent NADPH oxidase activity on cellular phenotype in human aortic VSMCs.

Regarding to the clinical application concluded from this study, since both in vitro MOVAS migration assay and the AS animal model in current study demonstrated that miR-155 had negative correlation with excessive VSMC migration and atherosclerotic plaques in the vessels wall, a 

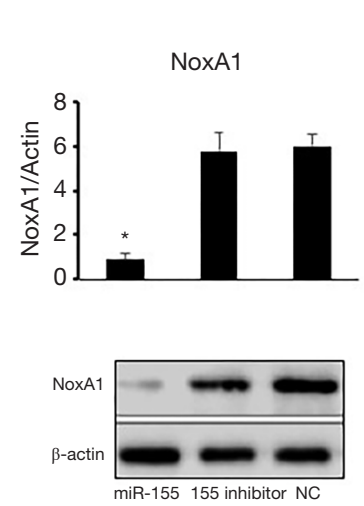

NADPH
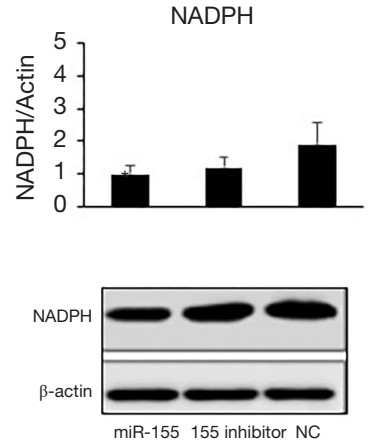

NoxA1 signaling pathway expression regulated by miR-155

Nox1
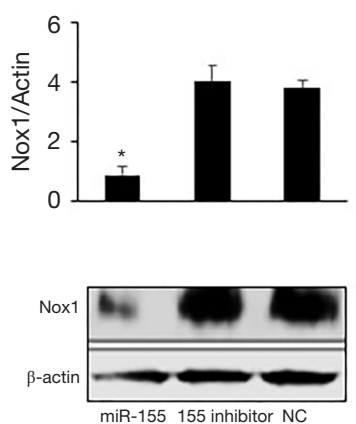

p47phox
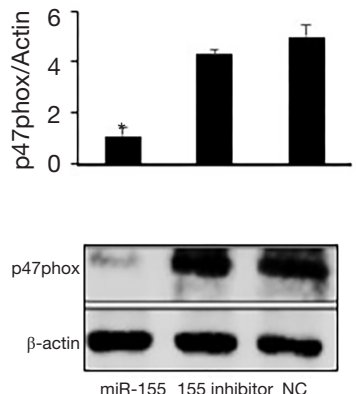

miR-155
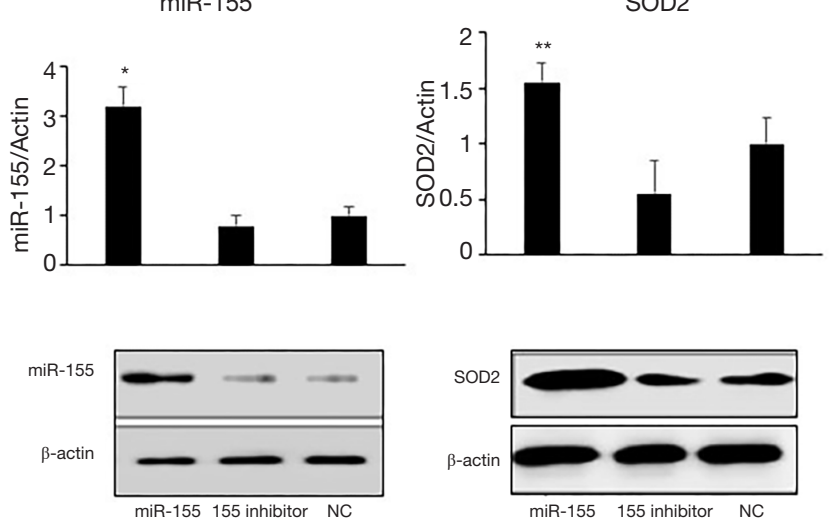

(*: miR-155 vs. 155 inhibitor and NC, $\mathrm{P}<0.05$ or $\mathrm{P}<0.01$; **: miR-155 vs. 155 inhibitor, $\mathrm{P}<0.05, \mathrm{n}=6$ )

Figure 6 Western blotting and corresponding real-time PCR of carotid artery specimens were performed to investigate the effect of miR155 on NoxA1-NADPH-p47phox complex and related signaling pathway. miR-155 mimic group showed similar trend as that in in vitro experiments, that was, significant inhibitory effect on NoxA1, Nox1 and p47phox expression while SOD2, the antioxidant was significantly higher than that of inhibitor group.

therapeutic strategy based on the functional role of miR155 in VSMC over proliferation-induced atherosclerosis maybe complementarily-synthesize miRNAs, which is a promising therapeutic tool due to the enhanced plaque stability and sustained efficacy after intravenous injection. This conception is also consistent with the clinical fact that miR-155 in PBMCs and plasma was significantly higher in hard plaque group in CAD patients (29). The conflicting results of miR-155 in the pathophysiology of atherosclerosis related vascular diseases indicate the complexity of this multifunctional molecule in regulation of cardiovascular remodeling induced by atherogenesis. The anticipated causes of this inconsistence might be different pathological stages of the diseases. Therefore, more comprehensive studies of underlying mechanisms of miR-155 involvement in CAD are needed (30).

Lastly, a notable limitation of our current study is that the importance of the miR-155/NoxA1/Nox1/ROS axis in atherosclerosis needs to be further verified by

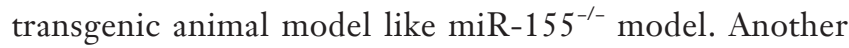
limitation is that beyond VSMC there are other types of cells contributing to the elevated plasma miR-155 levels in AS patients. In addition, the transfection of adenovirus containing miR-155 with in vivo VSMC is not stable and may need optimization accordingly.

\section{Acknowledgments}

Thanks for the technical support and experiments assistance of Central Lab of School of Medicine, Tongii University. Funding: This study was funded by the National Outstanding Youth Science Fund Project of National Natural Science Foundation of China (Grant NO. 81700316) and the Program of Outstanding Young Scientists of Tongji Hospital of Tongji University (HBRC1304, HBRC1801). 


\section{Footnote}

Reporting Checklist: The authors have completed the ARRIVE reporting checklist. Available at: http://dx.doi. org/10.21037/cdt-20-518

Data Sharing Statement: Available at http://dx.doi. org/10.21037/cdt-20-518

Peer Review File: Available at http://dx.doi.org/10.21037/ cdt-20-518

Conflicts of Interest: All authors have completed the ICMJE uniform disclosure form (available at http://dx.doi. org/10.21037/cdt-20-518). The authors have no conflicts of interest to declare.

Ethical Statement: All the authors are accountable for all aspects of the work in ensuring that questions related to the accuracy or integrity of any part of the work are appropriately investigated and resolved. Experiments were performed under a project license (NO.: 81700316) granted by institutional ethics committee board of Tongji Hospital affiliated to Tongji University, in compliance with Chinese national guidelines for the care and use of animals.

Open Access Statement: This is an Open Access article distributed in accordance with the Creative Commons Attribution-NonCommercial-NoDerivs 4.0 International License (CC BY-NC-ND 4.0), which permits the noncommercial replication and distribution of the article with the strict proviso that no changes or edits are made and the original work is properly cited (including links to both the formal publication through the relevant DOI and the license). See: https://creativecommons.org/licenses/by-nc-nd/4.0/.

\section{References}

1. GBD 2013 Mortality and Causes of Death Collaborators. Global, regional, and national age-sex specific all-cause and cause-specific mortality for 240 causes of death, 19902013: a systematic analysis for the Global Burden of Disease Study. Lancet 2013;385:117-71.

2. Zhou $\mathrm{M}$, Wang $\mathrm{H}$, Zhu J, et al. Cause-specific mortality for 240 causes in China during 1990-2013: a systematic subnational analysis for the Global Burden of Disease Study 2013. Lancet 2016;387:251-72.

3. Yan WW, Zhang KS, Duan QL, et al. Significantly reduced function of $\mathrm{T}$ cells in patients with acute arterial thrombosis. J Geriatr Cardiol 2015;12:287-93.

4. Yan W, Wen SW, Wang LM, et al. Comparison of cytokine expressions in acute myocardial infarction and stable angina stages of coronary artery disease. Int J Clin Exp Med 2015;8:18082-9.

5. Du F, Yu F, Wang YZ, et al. microRNA-155 deficiency results in decreased macrophage inflammation and attenuated atherogenesis in ApoE-/- mice. Arterioscler Thromb Vasc Biol 2014;34:759-67.

6. Babar IA, Czochor J, Steinmetz A, et al. Inhibition of hypoxia-induced miR-155 radiosensitizes hypoxic lung cance $r$ cells. Cancer Biol Ther 2011;12:908-14.

7. Niu XL, Madamanchi NR, Vendrov AE, et al. Nox activator 1: a potential target for modulation of vascular reactive oxygen species in atherosclerotic arteries. Circulation 2010;121:549-59.

8. Siu KL, Gao L, Cai H. Differential roles of protein complexes NOX1/NOXO1 and NOX2/p47phox in mediating endothelial redox responses to oscillatory and unidirectional laminar shear stress. J Biol Chem 2016;291:8653-62.

9. Streeter J, Schickling BM, Jiang S, et al. Phosphorylation of Nox1 regulates association with NoxA1 activation domain. Circ Res 2014; 115:911-8.

10. Li Y, Zhang GC, Wang XH, et al. Progression of atherosclerosis in ApoE-knockout mice fed on a high-fat diet. Eur Rev Med Pharmacol Sci 2016;20:3863-7.

11. Tallone T, Malin S, Samuelsson A, et al. A mouse model for adenovirus gene delivery. Proc Natl Acad Sci U S A 2001;98:7910-15.

12. Garrett KL, Anderson J. Colocalization of bFGF and the myogenic regulatory gene expression in dystrophic $\mathrm{mdx}$ muscle precursors and young myotubes in vivo. Dev Biol 1995; 169:596-608.

13. Glass CK, Witztum JL. Atherosclerosis. the road ahead. Cell 2001;104:503-16.

14. Chaabane C, Coen M, Bochaton-Piallat ML. Smooth muscle cell phenotypic switch: implications for foam cell formation. Curr Opin Lipidol 2014;25:374-9.

15. Moore KJ, Tabas I. Macrophages in the pathogenesis of atherosclerosis. Cell 2011;145:341-55.

16. Tuttolomondo A, Di Raimondo D, Pecoraro R, et al. Atherosclerosis as an inflammatory disease. Curr Pharm Des 2012;18:4266-88.

17. Hansson GK. Inflammation, atherosclerosis, and coronary artery disease. N Engl J Med 2005;352:1685-95.

18. Schroen B, Heymans S. Small but smart-microRNAs in 
the centre of inflammatory processes during cardiovascular diseases, the metabolic syndrome, and ageing. Cardiovasc Res 2012;93:605-13.

19. Baltimore D, Boldin MP, O'Connell RM, et al. MicroRNAs: new regulators of immune cell development and function. Nat Immunol 2008;9:839-45.

20. Lu LF, Thai TH, Calado DP, et al. Foxp3-dependent microRNA155 confers competitive fitness to regulatory $\mathrm{T}$ cells by targeting SOCS1 protein. Immunity 2009;30:80-91.

21. Sun HJ, Zhao MX, Liu TY, et al. Salusin- $\beta$ induces foam cell formation and monocyte adhesion in human vascular smooth muscle cells via miR155/NOX2/NFkB pathway. Sci Rep 2016;6:23596.

22. Zampetaki A, Dudek K, Mayr M. Oxidative stress in atherosclerosis: the role of microRNAs in arterial remodeling. Free Radic Biol Med 2013;64:69-77.

23. Brandes RP. Vascular functions of NADPH oxidases. Hypertension 2010;56:17-21.

24. Sun J, Ming L, Shang F, et al. Apocynin suppression of NADPH oxidase reverses the aging process in mesenchymal stem cells to promote osteogenesis and

Cite this article as: Tang $\mathrm{Y}$, Song $\mathrm{H}$, Shen $\mathrm{Y}$, Yao Y, Yu Y, Wei G, Long B, Yan W. MiR-155 acts as an inhibitory factor in atherosclerosis-associated arterial pathogenesis by downregulating NoxA1 related signaling pathway in $\mathrm{ApoE}^{-/}$mouse. Cardiovasc Diagn Ther 2021;11(1):1-13. doi: 10.21037/cdt-20518 increase bone mass. Sci Rep 2015;5:18572.

25. Li X, Kong D, Chen H, et al. miR-155 acts as antiinflammatory factor in atherosclerosis-associated foam cell formation by repressing calcium-regulated heat stable protein 1. Sci Rep 2016;6:21789.

26. Suh YA, Arnold RS, Lassegue B, et al. Cell transformation by the superoxide-generating oxidase Mox1. Nature 1999;401:79-82.

27. Leto TL, Lomax KJ, Volpp BD, et al. Cloning of a $67-\mathrm{kD}$ neutrophil oxidase factor with similarity to a noncatalytic region of p60c-src. Science 1990;248:727-30.

28. Kato M, Marumo M, Nakayama J, et al. The ROSgenerating oxidase Nox1 is required for epithelial restitution following colitis. Exp Anim 2016;65:197-205.

29. Zhu GF, Chu T, Ruan Z, et al. Inflammation-Related MicroRNAs Are Associated with Plaque Stability Calculated by IVUS in Coronary Heart Disease Patients. J Interv Cardiol 2019;2019:9723129.

30. Cao RY, Li Q, Miao Y, et al. The Emerging Role of MicroRNA-155 in Cardiovascular Diseases. Biomed Res Int 2016;2016:9869208. 